\title{
Erratum to: Persistent aryl hydrocarbon receptor inducers increase with altitude, and estrogen-like disrupters are low in soils of the Alps
}

\author{
Walkiria Levy • Bernhard Henkelmann • Silke Bernhöft • Toine Bovee • \\ Franz Buegger • Gert Jakobi • Manfred Kirchner • Rodolfo Bassan • \\ Norbert Kräuchi • Wolfgang Moche • Ivo Offenthaler • Primoz Simončič • \\ Peter Weiss • Karl-Werner Schramm
}

Published online: 21 October 2014

(C) Springer-Verlag Berlin Heidelberg 2014

\section{Erratum to: Environ Sci Pollut Res DOI 10.1007/s11356-010-0361-8}

In the following paper the units of the yeast estrogen bioassay were by mistake changed from the real unit: "ng 17ß-estradiol EQ g ${ }^{-1}$ dry soil" to "pg 17ß-estradiol EQ $\mathrm{g}^{-1}$ dry soil".

This causes the following important changes in the text:

The online version of the original article can be found at http://dx.doi.org/ 10.1007/s11356-010-0361-8.

\footnotetext{
W. Levy $(\bowtie) \cdot$ B. Henkelmann · S. Bernhöft · G. Jakobi $\cdot$

M. Kirchner $\cdot$ K.-W. Schramm

Institute of Ecological Chemistry, Helmholtz Zentrum,

München-German Research Center for Environmental Health,

Ingolstädter Landstrasse 1,

85764 Neuherberg, Germany

e-mail: walkiria.levy@helmholtz-muenchen.de

K.-W. Schramm $(\bowtie)$

Department für Biowissenschaftliche Grundlagen,

TUM-Technische Universität München,

Weihenstephaner Steig 23,

85350 Freising, Germany

e-mail: schramm@wzw.tum.de

T. Bovee

Rikilt, Institute of Food Safety, Wageningen University and Research Center,

6700 AE Wageningen, The Netherlands

e-mail: Toine.Bovee@wur.nl

F. Buegger

Helmholtz Zentrum München-German Research

Center for Environmental Health, Institute for Soil Ecology,

Ingolstädter Landstrasse 1,

85764 Neuherberg, Germany

e-mail: buegger@helmholtz-muenchen.de
}

\begin{abstract}
Page 2: $0.37 \pm 0.12$ ng $17 \beta$-estradiol EQ g ${ }^{-1}$ dry soil Instead of: $0.37 \pm 0.12 \mathrm{pg}^{17 \beta}$-estradiol EQ g ${ }^{-1}$ dry soil

Conclusion Low bioassay responses with a higher relative amount of ER disrupters than AhR inducers were detected, indicating the higher abundance of estrogen-like than persistent dioxin-like compounds in these forested areas.
\end{abstract}

\author{
R. Bassan \\ Regional Agency for Environmental Prevention \\ and Protection of Veneto, \\ Via Matteotti 27, \\ 35137 Padova, Italy \\ e-mail: r.basssan@arpa.veneto.it \\ N. Kräuchi \\ WSL-Swiss Federal Institute for Forest, \\ Snow and Landscape Research, \\ Zürcherstrasse 111, \\ 8903 Birmensdorf, Switzerland \\ e-mail:kraeuchi@wsl.ch \\ W. Moche $\cdot$ I. Offenthaler $\cdot$ P. Weiss \\ Umweltbundesamt $\mathrm{GmbH}$, \\ Spittelauer Lände 5, \\ 1090 Wien, Austria \\ W. Moche \\ e-mail: wolfgang.moche@umweltbundesamt.at \\ P. Simončič \\ Slovenian Forestry Institute, \\ Več pot 2, \\ 1000 Ljubljana, Slovenia \\ e-mail: primoz.simoncic@gozdis.si
}


Instead of: Low bioassay responses with a higher relative amount of AhR inducers than ER disrupters were detected, indicating the higher abundance of persistent dioxin-like than estrogen-like compounds in these forested areas.

\section{Materials and Methods}

2.3 Genetically modified yeast bioassay

Page 4: The LOD is $0.03 \mathrm{ng} 173$-estradiol EQ g ${ }^{-1}$ dry soil, whereas the LOQ is $0.05 \mathrm{ng} 173$-estradiol EQ $\mathrm{g}^{-1}$ dry soil. Instead of: The LOD is 0.03 pg 17ß-estradiol EQ $\mathrm{g}^{-1}$ dry soil, whereas the LOQ is 0.05 pg $17 ß$-estradiol EQ g ${ }^{-1}$ dry soil.

\section{Results}

Table 1:

Estrogenic activity (ng 17ß-estradiol EQ g ${ }^{-1}$ dry soil)

LOQ Estrogenicity $0.05 \mathrm{ng} 173-\mathrm{E}$ EQ g ${ }^{-1}$ dry soil

Estrogenicity (ng 17ß-E EQ g ${ }^{-1}$ dry soil)

Instead of:

Estrogenic activity (pg 17ß-estradiol EQ $\mathrm{g}^{-1}$ dry soil)

LOQ Estrogenicity 0.05 pg 17ß-E EQ g ${ }^{-1}$ dry soil

Estrogenicity (pg 17ß-E EQ g ${ }^{-1}$ dry soil)

\subsection{Yeast-based bioassay}

Page $7: \ldots$ whereas the mean values obtained in the present study are $0.37 \pm 0.12 \mathrm{ng} 17 ß$-estradiol EQ g ${ }^{-1}$ dry soil...

Instead of:

$\therefore$..whereas the mean values obtained in the present study are $0.37 \pm 0.12$ pg $17 \beta$-estradiol EQ $\mathrm{g}^{-1}$ dry soil...
... but the higher relative amounts of ER disrupters than AhR inducers is obvious, pointing out the higher abundance of estrogen-like inducers than persistent dioxin-like compounds ... Instead of:... but the higher relative amounts of AhR inducers than ER disrupters is obvious, pointing out the higher abundance of persistent dioxin-like compounds than estrogen-like inducers ...

"...whereas the Alpine area had a ratio of around $\mathbf{0 . 0 1 6}$." Instead of: "...whereas the Alpine area had a ratio of around 16.”

\section{Conclusions}

Finally, ER disrupters were found at low concentrations in these Alpine areas.

This statement can only be verified in function of future research in this area with environmental soil samples. The current research is most based on environmental sediment matrices that are in the same range or clearly above the here presented values. However, based on the available literature regarding soils, the only work found based in the study of estrogenicity of environmental soils (Xiao et al., 2006) pointed out noticeably lower values than the here obtained soil estrogenicity values.

Instead of:

Finally, ER disrupters were found at low concentrations in these Alpine areas. 